\title{
An Improved Technique for Increasing the Accuracy of Joint-to-Ground Distance Tracking in Kinect v2 for Foot-Off and Foot Contact Detection
}

\author{
Amin Amini ${ }^{1 *}$ and Konstantinos Banitsas ${ }^{2}$ \\ College of Engineering Design and Physical Sciences, Department of Electronics \\ and Computer Engineering, Brunel University London; \\ amin.amini@brunel.ac.uk ${ }^{1}$ \\ konstantinos.banitsas@brunel.ac.uk² \\ * Correspondence: amin.amini@brunel.ac.uk; Tel.: +44-1223940349
}

\section{An Improved Technique for Increasing the Accuracy of Joint-to-Ground Distance Tracking in Kinect v2 for Foot-Off and Foot Contact Detection}

\footnotetext{
Abstract: The Kinect sensor has been widely used in different applications such as rehabilitation and gait analysis. Whilst Kinect v2 was released with several improvements over its predecessor, it still incorporates depth-map intrinsic inaccuracies. This results in
} 
inconsistencies in skeletal-data acquisition, especially in joint localization and distance-toground tracking with respect to the Kinect's 3-D Cartesian coordinate reference point. This research proposes a correction technique based on the two-point linear equation derived from the information gathered from different subjects' skeletal data and data regression analysis to compensate the inaccuracies in joint-to-ground data collection. The research also proposes a new footsteps detection method based on skeletal data and plane detection techniques that calculates a footstep by using the ankle's Euclidean distance from the floor, regardless of the subject's distance from the camera. The results show that after the correction technique was applied, data acquisition proved to be consistent and more accurate within a distance range of 1.6 to 2.9 metres from the Kinect camera, regardless of the subject's location to the camera's reference point. Moreover, the inconsistency of joint data read by the Kinect was reduced from $25.69 \%$ to $5.25 \%$ and the footsteps detection accuracy increased from $42.85 \%$ to $79.76 \%$ on average for both legs.

Keywords: gait cycle; pattern recognition; footsteps detection; depth measurement; Kinect v2; FOG; machine vision.

\section{Disclosure statement:}

The authors report no conflict of interest.

\section{Introduction}

Detecting footsteps plays an important role in gait cycle analysis and rehabilitation purposes, as many diseases feature physical symptoms, especially gait disorders. Having an unobtrusive gait detection system can significantly improve the accuracy of footsteps analysis, as due to the nature of the case, on-body sensors can sometimes be problematic and have a direct effect on the gait performance and behaviour. Different methods have been used in extracting accurate information related to footsteps detection such as pressure-based mapping, in which foot contacts and foot-offs can be detected based on the variance in pressure in different areas of a foot sole (Ghoussayni et al., 2004; Hreljac et al., 2000), inertial-based sensing using different wearable sensors attached to the body (Auvinet et al., 2015; Amini Maghsoud Bigy et al., 2015; Bundle et al., 2015b), instrumented treadmills (Bundle et al., 2015a; ItemGlatthorn et al., 2016; Wearing et al., 2013) and computer vision systems (Ijaz et al., 2012; Kwolek et al., 2014). Most of the common methods in gait analysis, especially footsteps 
detection, are obtrusive and expensive to implement.

The Microsoft Kinect sensor is an ideal sensing device to detect footsteps as it does not require any devices or sensors to be attached to the subject's body. Since the introduction of the Microsoft Kinect sensor, many studies have been conducted based on the Kinect camera with regards to gait performance analysis (Amini et al., 2017; Amini Maghsoud Bigy et al., 2015; Clark et al., 2013; Gabel et al., 2012; Galna et al., 2014; Procházka et al., 2015; E. E. Stone \& Skubic, 2013). Nonetheless, Kinect skeletal-based detection of footsteps, in particular, is a challenging feat due to Kinect's margin of error, especially for lower extremities (Galna et al., 2014). Moreover, Kinect v2 in particular, lacks built-in features available in the first iteration of Kinect such as Joint Filtering that could compensate the sensor's erroneous data acquisition to some extent. This led to some innovation methods to compensate the Kinect's aforementioned inaccuracies. For instance, in (Ahmed et al., 2015), a new Kinect-based gait recognition technique was used in which human gait signature was analysed using spatio-temporal changes in different skeletal joints' angles. Having a joint relative angle for stride detection eliminates the Kinect sensor's inaccuracies due to the subject's direction or distance from the camera (Clark et al., 2013; Corti et al., 2016; E. Stone \& Skubic, 2011; Yang et al., 2015). The research used the spine joint as the reference point as its relative 3-D coordination remains almost stationary during a gait cycle. Nevertheless, by employing such a technique, foot contact and foot-off phases will not be directly detected, but instead be estimated based on the distance and angle of skeletal joints.

In (Auvinet et al., 2015), heel-strikes were estimated by calculating the distance between knees' joint centre along the longitudinal walking axis. To eliminate the Kinect depth-map inaccuracies in localising joints according to a subject's distance from the sensor during a gait cycle (especially for foot contact detection (Galna et al., 2014)), knee height was estimated based on anthropometric data. In another attempt by (Geerse et al., 2015), a series of 4 Kinect v2 sensors were placed in pre-determined locations to compensate each Kinect's depth inaccuracies in farther distances and have an overall wider range of coverage. This method provided promising results but at the expense of using an array of Kinect v2 cameras that required precise alignment between each sensor and increased the cost considerably. (Xu et al., 2015) used a Kinect camera mounted on a treadmill whilst the subject performed gait cycles in order to keep the subject's distance to the camera consistent. In (Sun et al., 2014) a rather innovative technique was employed by putting the subject in a Kinect-mounted cart to keep the subject's distance from the Kinect consistent whilst walking. 
Most of the aforementioned methods can affect gait performance accuracy as they influence the subject's natural way of walking, whilst others require expensive or difficult-toimplement improvisations. More importantly, some gait performance analysis and stepdetection scenarios such as detecting Freezing of Gait (FOG) in People with Parkinson's $(\mathrm{PwP})$, mandate precise data reading; minor inaccuracies in joint localisation, will render the entire data reading unusable.

This research analyses the data gathered from different subjects in different conditions in order to correct the Kinect's joint-to-ground distance data reading issues according to camera's 3-D Cartesian $\mathrm{Z}$ axis. It also proposes a new technique in footsteps detection using skeletal data and plane detection techniques.

\section{Methods}

Eleven healthy subjects (Table 1) participated the trial in which they were asked to walk in pre-defined paths whilst their skeletal data being captured and analysed by the Kinect camera.

\section{Table 1}

Subjects were asked to walk in pre-defined paths: 12 per subject, by walking towards the camera whilst having the Kinect camera's angle ranging from 0 to $45(0,10,22$ and 45) degrees to the ground at Kinect's height of $0.65,1$ and 1.57 metres to the ground in order to identify any possible differentiation in results based on the camera angle and height factors, whilst their skeletal data was captured and analysed by the Kinect camera. The software was written in C\# using Kinect for Windows SDK version 2.0.1410.19000. For simplicity, the paper only shows the subjects' left ankle throughout the figures.

For further evaluation of the system, its outputs had to be compared for accuracy to a system considered as a golden standard. A series of eight synchronised Vicon T10 motion capture cameras providing full room coverage were used alongside the Kinect v2 camera. The test subjects were asked to walk in a pre-determined path while their skeletal joints were being monitored by both the Kinect v2 and Vicon cameras.

For each test, we recorded the first 10 seconds of the subject walking for our system to calculate the correction algorithm. Subjects were asked to walk towards the Kinect from the distance of 4.33 metres to 1.38 metre to the Kinect camera. The collected data were used 
in the correction algorithm to rectify the Kinect's intrinsic inaccuracies mentioned previously in order to provide more accurate subject's joint-to-ground data. Consequently, the results were used to detect subjects' footstep including foot-offs and foot contacts directly based on their ankles' distance to the ground.

\section{a) Testing Environment}

A testing environment was used to carry out the trials as below:

\section{Figure 1}

Error! Reference source not found.a shows the process of collaborating virtual markers attached to a subject for Vicon cameras where a subject in a T-pose for her ankles height, as well as her joints' position to be calculated by both Vicon and Kinect systems. The Vicon cameras and Kinect v2 captured each session simultaneously while the frame rate of the recorded data from the Vicon cameras was lowered down to match the Kinect v2 at approximately 30 frames per second.

Error! Reference source not found.b and Error! Reference source not found.c show real-time 3D data representation of both the Vicon cameras and Kinect v2, respectively.

Note that the motion capture suit used in the experiment were intended for the Vicon cameras only as the Kinect v2 does not require any special clothing for its skeletal detection system to function. Subjects were also asked to wear normal clothing after the initial ankle measurement phase while their movements were being recorded by Kinect v2 in different situations (camera heights and angles).

\section{Figure 2}

\section{b) Correction Algorithm}


In order to assist the system in correcting the inconsistencies in the footstep detection algorithm, the relation between the subject's ankles Z-axis to the Kinect's sensor and subject's ankles distance to the ground was examined.

The first 10 seconds of the Kinect skeletal data, including the subject's ankles height to the ground and subject's ankles distance to the camera for each test subject were recorded and filtered to include only standstill positions in order to acquire a baseline of calculated ankles' height to the ground. The data was then run through a regression analysis by the developed system to adjust the depth-map correction algorithm. A two-point linear equation was used to estimate the correct ankle's height to the floor at any given time based on the subject's ankle Z-axis distance from the camera.

$$
\frac{y-y_{1}}{z-z_{1}}=\frac{y_{2}-y_{1}}{z_{2}-z_{1}}(1)
$$

The above equation is correlation between a joint's Z-axis and Y-axis where $z$ is the ankle's Z-axis distance to the camera and $\boldsymbol{y}$ is ankle's 3-D Euclidean distance to the ground at any given time. After an initial 10 seconds data recording of all trials for the correction algorithm analysis, the value of $\boldsymbol{y}$ in equation (1) was calculated as follows:

$$
y=x \times 0.013 \pm 0.05
$$

Equation (2) is derived from simplifying and substituting numbers collected from the initial 10 seconds of data recording in equation (1).

The following equation was then used to correct the depth map stream data reading. By applying the corrected y value yielded from Equation (2) to the following equation, the true value of the $\boldsymbol{d}$ can be calculated as follows:

$$
\hat{d}_{i}=d_{i}+\left(l-z_{i}\right) \frac{y_{i}}{z_{i}}(3)
$$


where $\widehat{\boldsymbol{d}}_{\boldsymbol{i}}$ is the corrected estimate of ankle's distance to ground for the $i$ th stride, $\boldsymbol{d}_{\boldsymbol{i}}$ is the Kinect's measured distance of the subject's ankle yielded from equation $(5), \boldsymbol{z}_{\boldsymbol{i}}$ is the ankle's Z-axis distance from the Kinect, and $\boldsymbol{y}_{\boldsymbol{i}}$ is the correction factor for the ith stride. $\boldsymbol{l}$ is the maximum visible distance of Kinect in $\mathrm{Z}$-axis and remains consistent. All the values are in meters as detected by the Kinect's depth sensor.

\section{c) Footsteps Detection}

Kinect skeleton data were used to calculate ankles' joint 3-D Cartesian coordinate locations. Once a joint was localised using Kinect skeleton data, the surface floor is determined based on the scalar equation of planes.

$$
A x+B y+C z+D=0(4)
$$

Where $\boldsymbol{A}, \boldsymbol{B}$ and $\boldsymbol{C}$ are the components of a normal vector that is perpendicular to any vector in a given plane that are determined by the Kinect once at least a subject is present in a scene and $\boldsymbol{D}$ is the height of the Kinect from the levelled floor.

As Figure . demonstrates, ankles and feet are the only Kinect-discoverable joints having significant displacement changes in correlation with gait cycles whilst retaining least errors compared to the movements of upper human extremities. As mentioned previously, although some studies including (Amini et al., 2017) discussed methods based on joints' anterior and posterior displacement changes, this proposed method can be used in scenarios that joints' anterior and posterior displacement changes have little correlation with gait cycles such as FOG incidents in PwP. As observed during the study, Kinect's detection of ankles was less susceptible to noise and inaccuracies compared to feet. A filter was applied to the signal in order to acquire a baseline of subjects' ankles and feet height only in a stand-still position. An average inaccuracy was calculated based on the deviation between the estimated ankles height and the actual ankles height. Moreover, the inaccuracy between the estimated feet height and the actual feet height was also calculated. The result showed $25.69 \%$ and $44.43 \%$ inaccuracies for all subjects' ankles and feet, respectively. Moreover, according to 
(Wang et al., 2015), it was concluded that in the lower extremities, a subject's feet are more susceptible to noise due to their close distance to large planar surfaces. Thus, subjects' ankles were chosen to evaluate and track footsteps.

\section{Figure 3}

A foot-off event is considered to have occurred when a foot's ankle 3-D Euclidean distance from the floor has increased to more than a particular threshold based on the actual ankle's height. Consequently, a foot contact event is triggered when the ankle 3-D Euclidean distance from the floor of the same foot has returned to its original value in a time period of more than 250 milliseconds. The $250 \mathrm{~ms}$ timing threshold was set to avoid the false positives flag ups due to the Kinect inconsistencies and noise. The Euclidean distance of an ankle's 3D Cartesian coordinate location from the ground can be yielded as follows:

$$
d=\frac{A x+B y+C z+D}{\sqrt{A^{2}+B^{2}+C^{2}}}
$$

Where $\boldsymbol{x}, \boldsymbol{y}$ and $\boldsymbol{z}$ are the 3-D coordinates' location of a joint. A, B, C and D are the same as in equation (4).

Figure . demonstrates the subject's footsteps detection process including foot-offs and foot contacts. The algorithm loops at approximately 30 frames per second whilst the Kinect camera is tracking the subject's movements.

\section{Figure 4}

\section{Figure 1}


Figure 2. shows the calculated height of a subject's ankle using Equation 5 (before the correction was applied). The algorithm checks whether the subject's ankle height value is greater than the actual size of the subject's ankle. If the result was true, the algorithm then starts a timer to calculate the swing time. As soon as the initial condition becomes false, the algorithm then stops the timer and increment the number of footstep for each foot. Subjects were asked to move towards the Kinect camera and remain still at different distances from the Kinect's lens optical centre. The Kinect RGB feed was then aligned with its depth/skeleton feed using the Kinect coordinate mapping technique. The figure demonstrates the Kinect's inaccuracies in detecting a subject's ankle distance to the ground. Even in standstill posture, as the subject's Z coordinates (depth) to the Kinect's sensor changes, the Kinect reads different value of the subject's ankle distance to the ground.

\section{Figure 2}

Figure 3. Panel (a) shows a subject's left ankle height to the ground at different distances from the Kinect camera while the subject is walking towards the Kinect camera before the correction was applied. The peaks in Panel (a) show the steps taken by the subject. The dotted line represents the subject's actual ankle height in a stand still position. Panel (b) shows the same subject's ankle Z-axis distance from the Kinect camera during the same walking session.

It was observed that not only the subject's ankles height was changing in accordance to its Zaxis distance from Kinect camera, but also the calculated distance of the ankle from the floor was not consistent even in a stand-still position. The study showed that as the subject's ankle Z-axis distance from the Kinect camera decreases, the subject's calculated ankle height to the ground also decreases.

\section{Results}

Figure 4. shows that after the correction technique based on the two-point linear equation was applied, the data reading proved to be consistent, and the calculated ankle's height is closer to the actual measured height (in the most commonly used range of 1.6 to 2.9 metres from the Kinect camera) regardless of the subject's location to the camera. 


\section{Figure 4}

Figure 5. Panel (a) compares the subject's left ankle height to the ground during gait analysis in both scenarios (w/ and w/o the correction being applied). The dotted line represents the subject's actual ankle height in stand still position. Panel (b) shows the subject's left ankle height to the ground and its Z-axis distance to the Kinect camera. Panel (c) demonstrates the subject's walking path (t1) as the subject moved towards the Kinect camera and (t2) as the subject returned to initial previous location.

\section{Figure 5}

Figure 5. Panel (c) shows a subject's walking path towards the Kinect camera. The walking path consisted of two phases (t1) walking towards the Kinect camera and (t2) moving away from the Kinect camera. The subject was at 45 degrees in reference to the Kinect camera. Figure 7Panel (b) shows the subject's left ankle height to the ground and its Z-axis distance to the Kinect camera. The result showed that the ankle's angle to the Kinect's camera references point does not affect the data reading and the correction algorithm. Panel (a) compares the subject's left ankle height to the ground during gait analysis in both scenarios (with and without the correction being applied). The dotted line represents the subject's actual ankle height in stand still position.

It was observed that the Kinect's height to the ground did not have any impact on the data collection whereas its angle to the floor proved to have a statistically significant effect on the data collection and readings. While the Kinect's data collection and consequently the proposed correction algorithm accuracy was at their highest when the Kinect's angle to the floor was within the range of $15 \pm 3$ and $45 \pm 3$ degrees, angles higher/lower than this range proved to be problematic and inaccurate. A possible explanation would be the effect of the Kinect's limited field of view on covering subjects' joints and detecting floor plane during the entire gait cycle.

Figure 6. illustrates the effect of different Kinect's angle to ground on its data collection accuracy and the proposed correction algorithm. The corrected data was then used to calculate the gait characteristics and the number of footsteps. 


\section{Figure 6}

Table 2 presents the Kinect v2 inaccuracies comparison for both before and after the correction algorithm was applied. It is clear that the margin of error varies between different subjects, which can be explained by different Kinect behaviour in data collection based on subjects' different shoes and trousers types, as well as colours and materials. Nonetheless, the difference between subject's actual and calculated ankle height deviation for all subjects fall under the estimated margin of this study.

\section{Table 2}

\section{Table 3}

Table 3 shows the correction algorithm effect on the improvement of the accuracy of Kinect $\mathrm{v} 2$ in detecting foot contacts using the proposed method. It shows that the correction algorithm improved the accuracy by more than $37 \%$ and $36 \%$ on average, for right and left ankles, respectively.

\section{Discussion}

A correction algorithm was applied to subjects' ankles distance to the floor in order to compensate for the Kinect's v2 inconsistencies in joints' localisation, which ultimately made footsteps detection based on skeletal data and plane detection techniques possible. The initial ankle's height data reading inaccuracies were decreased after the correction algorithm was applied from $25.72 \%$ and $25.66 \%$ (R/L ankle) to about $5.59 \%$ and $4.91 \%$ on average, amongst all subjects. Therefore, resulting in greater accuracy in footstep detection from the original $42.06 \%$ and $43.65 \%$, to $79.37 \%$ and $80.16 \%$ on average, for right and left ankles, respectively, amongst all subjects. It was studied that the effective range for the correction algorithm was between 1.6 to 2.9 metres from the Kinect camera; in which before and after this range, the data reading inaccuracies returned to the original values. Moreover, although the Kinect's height did not affect the data reading, the camera's angle had a statistically significant effect: it was observed that whilst the camera's angle to the floor facing downward is within the range of $15 \pm 3$ and $45 \pm 3$ degrees, the data were also at their highest accuracy. 
This can be due to the fact that angles lower that $15 \pm 3$ and higher than $45 \pm 3$ degrees cannot cover most of the subject's joints and detecting floor plane in a frame due to the Kinect's limited field of view.

\section{Conclusion}

The results of this research improve the joint 3-D localisation at different distances and the accuracy of footsteps detection and using only a single Kinect v2 sensor, which can be used in various gait analysis researches. As a next step, the findings in this research will be used in research projects addressing the detection of FOG in PwP, which requires a higher accuracy in data reading for footstep detection, due to the nature of the symptom. Moreover, future research will investigate the Kinect v2 sensor's shortcomings in detecting gait cycle's swing phase, and initial contact in a stance phase, based on skeleton data and plane detection techniques.

\section{Word Count: 3445}

\section{References}

Ahmed, F., Paul, P. P., \& Gavrilova, M. L. (2015). Kinect-Based Gait Recognition Using Sequences of the Most Relevant Joint Relative Angles, 23(2), 147-156.

Amini, A., Banitsas, K., \& Hosseinzadeh, S. (2017). A new technique for foot-off and foot contact detection in a gait cycle based on the knee joint angle using microsoft kinect v2. In 2017 IEEE EMBS International Conference on Biomedical \& Health Informatics (BHI) (pp. 153-156). Orlando: IEEE. https://doi.org/10.1109/BHI.2017.7897228

Auvinet, E., Multon, F., Aubin, C. E., Meunier, J., \& Raison, M. (2015). Detection of gait cycles in treadmill walking using a Kinect. Gait and Posture, 41(2), 722-725. https://doi.org/10.1016/j.gaitpost.2014.08.006

Bigy, A. A. M., Banitsas, K., Badii, A., \& Cosmas, J. (2015). Recognition of Postures and Freezing of Gait in Parkinson's Disease Patients Using Microsoft Kinect Sensor. 7th Annual International IEEE EMBS Conference on Neural Engineering, 731-734. https://doi.org/10.1109/NER.2015.7146727

Bundle, M. W., Powell, M. O., \& Ryan, L. J. (2015b). Design and testing of a high-speed treadmill to measure ground reaction forces at the limit of human gait. Medical Engineering and Physics, 
37(9), 892-897. https://doi.org/10.1016/j.medengphy.2015.04.009

Bundle, M. W., Powell, M. O., \& Ryan, L. J. (2015a). Design and testing of a high-speed treadmill to measure ground reaction forces at the limit of human gait. Medical Engineering and Physics, 37(9), 892-897. https://doi.org/10.1016/j.medengphy.2015.04.009

Clark, R. A., Bower, K. J., Mentiplay, B. F., Paterson, K., \& Pua, Y. H. (2013). Concurrent validity of the Microsoft Kinect for assessment of spatiotemporal gait variables. Journal of Biomechanics, 46(15), 2722-2725. https://doi.org/10.1016/j.jbiomech.2013.08.011

Corti, A., Giancola, S., Mainetti, G., \& Sala, R. (2016). A metrological characterization of the Kinect V2 time-of-flight camera. Robotics and Autonomous Systems, 75, 584-594. https://doi.org/10.1016/j.robot.2015.09.024

Gabel, M., Gilad-Bachrach, R., Renshaw, E., \& Schuster, A. (2012). Full Body Gait Analysis with Kinect*. In 2012 Annual International Conference of the IEEE Engineering in Medicine and Biology Society (pp. 1964-1967). San Diego, CA: IEEE.

Galna, B., Barry, G., Jackson, D., Mhiripiri, D., Olivier, P., \& Rochester, L. (2014). Accuracy of the Microsoft Kinect sensor for measuring movement in people with Parkinson's disease. Gait $\{\&\}$ Posture, 39(4), 1062-1068. https://doi.org/10.1016/j.gaitpost.2014.01.008

Geerse, D. J., Coolen, B. H., \& Roerdink, M. (2015). Kinematic validation of a multi-Kinect v2 instrumented 10-meter walkway for quantitative gait assessments. PLoS ONE, 10(10), 1-15. https://doi.org/10.1371/journal.pone.0139913

Ghoussayni, S., Stevens, C., Durham, S., \& Ewins, D. (2004). Assessment and validation of a simple automated method for the detection of gait events and intervals. Gait and Posture, 20(3), 266272. https://doi.org/10.1016/j.gaitpost.2003.10.001

Hreljac, A., \& Marshall, R. N. (2000). Algorithms to determine event timing during normal walking using kinematic data. Journal of Biomechanics, 33(6), 783-786. https://doi.org/10.1016/S00219290(00)00014-2

Ijaz, A., Gibbs, S., Abboud, R., Wang, W., Ming, D., \& Wan, B. (2012). Analysis of knee joint kinematics during walking in patients with cerebral palsy through human motion capture and gait model-based measurement. Proceedings of IEEE International Conference on Virtual Environments, Human-Computer Interfaces, and Measurement Systems, VECIMS, 196-199. https://doi.org/10.1109/VECIMS.2012.6273181

Item-Glatthorn, J. F., Casartelli, N. C., \& Maffiuletti, N. A. (2016). Reproducibility of gait parameters at different surface inclinations and speeds using an instrumented treadmill system. Gait $\{\&\}$ 
Posture, 44, 259-264. https://doi.org/10.1016/j.gaitpost.2015.12.037

Kwolek, B., Krzeszowski, T., \& Michalczuk, A. (2014). 3D Gait Recognition Using Spatio-Temporal. Intelligent Information and Database Systems, 6th Asian Conference, 595-604.

Procházka, A., Vyšata, O., Vališ, M., `Tupa, O., Schätz, M., \& Ma`rík, V. (2015). Use of the image and depth sensors of the Microsoft Kinect for the detection of gait disorders. Neural Computing and Applications, 26(7), 1621-1629. https://doi.org/10.1007/s00521-015-1827-x

Stone, E. E., \& Skubic, M. (2013). Unobtrusive, continuous, in-home gait measurement using the microsoft kinect. IEEE Transactions on Biomedical Engineering, 60(10), 2925-2932. https://doi.org/10.1109/TBME.2013.2266341

Stone, E., \& Skubic, M. (2011). Evaluation of an inexpensive depth camera for in-home gait assessment. Journal of Ambient Intelligence and Smart Environments, 3(4), 349-361. https://doi.org/10.3233/AIS-2011-0124

Sun, B., Liu, X., Wu, X., \& Wang, H. (2014). Human gait modeling and gait analysis based on Kinect. Proceedings - IEEE International Conference on Robotics and Automation, 3173-3178. https://doi.org/10.1109/ICRA.2014.6907315

Wang, Q., Wang, Q., Kurillo, G., Ofli, F., \& Bajcsy, R. (2015). Evaluation of Pose Tracking Accuracy in the First and Second Generations of Microsoft Kinect. In International Conference on Healthcare Informatics (pp. 380-389). Dallas, TX: IEEE. https://doi.org/10.1109/ICHI.2015.54

Wearing, S. C., Reed, L. F., \& Urry, S. R. (2013). Agreement between temporal and spatial gait parameters from an instrumented walkway and treadmill system at matched walking speed. Gait and Posture, 38(3), 380-384. https://doi.org/10.1016/j.gaitpost.2012.12.017

Xu, X., McGorry, R. W., Chou, L. S., hua Lin, J., \& chi Chang, C. (2015). Accuracy of the Microsoft Kinect $^{\mathrm{TM}}$ for measuring gait parameters during treadmill walking. Gait and Posture, 42(2), 145151. https://doi.org/10.1016/j.gaitpost.2015.05.002

Yang, L., Zhang, L., Dong, H., Alelaiwi, A., \& Saddik, A. (2015). Evaluating and improving the depth accuracy of Kinect for Windows v2. IEEE Sensors Journal, 15(8), 4275-4285. https://doi.org/10.1109/JSEN.2015.2416651 